\title{
Semi-Blind Interference Prediction in Wireless Networks
}

\author{
Mahin K. Atiq, Udo Schilcher, Jorge F. Schmidt, and Christian Bettstetter \\ Institute of Networked and Embedded Systems, University of Klagenfurt, Austria \\ Email: firstname.lastname@aau.at
}

\begin{abstract}
Our research investigates the concept of interference prediction as an unprecedented approach for interference management and medium access in wireless networks. This paper is a first step in this direction: it proposes and evaluates a simple interference prediction technique that is based on low-complexity learning. Nodes predict the interference situation they expect to experience in the near future and select the most favorable time slot to start the transmission of a multislot message. The performance gain is evaluated in a small-scale fading environment in terms of link outage and delay against random slot selection. Simulation results show that interference prediction is a promising building block for wireless systems. Additional studies are needed to explore advanced techniques and assess their feasibility.
\end{abstract}

\section{KEYWORDS}

Wireless networks; Interference prediction; Interference modeling

\section{ACM Reference format:}

Mahin K. Atiq, Udo Schilcher, Jorge F. Schmidt, and Christian Bettstetter. 2017. Semi-Blind Interference Prediction in Wireless Networks. In Proceedings of MSWiM '17, Miami, FL, USA, November 21-25, 2017, 5 pages. https://doi.org/10.1145/3127540.3127579

\section{INTRODUCTION}

Interference is a stochastic signal that disturbs the reception of an intended signal at a node in a network. Despite advances in understanding the space-time dynamics of interference in wireless networks $[4,6,13,16]$, researchers have not progressed from modeling to designing so far. In other words, an open issue is: How can knowledge about interference dynamics be harnessed to improve communication - maybe in a similar way as we harness knowledge about channel dynamics (e.g., coherence time, decorrelation distances) in today's technologies? Addressing this question may open a new field with potentially high impact: the science of interference prediction. This paper is our first step in this direction. We propose a simple interference predictor and analyze its potential, thus going beyond using interference dynamics as a sole analysis tool.

We focus on interference dynamics arising from nodes' traffic patterns. Different nodes send, in general, at different time instances and with different sending durations. Such traffic patterns can be acquired in practice by monitoring the activity of nodes. Three contributions are made: First, we design an interference predictor

Permission to make digital or hard copies of part or all of this work for personal or classroom use is granted without fee provided that copies are not made or distributed for profit or commercial advantage and that copies bear this notice and the full citation on the first page. Copyrights for third-party components of this work must be honored.

For all other uses, contact the owner/author(s).

MSWiM '17, November 21-25, 2017, Miami, FL, USA

(c) 2017 Copyright held by the owner/author(s).

ACM ISBN 978-1-4503-5162-1/17/11.

https://doi.org/10.1145/3127540.3127579 based on learning the transmission behavior of interfering nodes. Second, we propose a transmission slot selection technique based on interference prediction for slotted medium access. Third, we assess the gains in outage and delay resulting from using such slot selection in a Poisson network with small-scale fading.

Based on the fact that interference is dominated by the closest interferers [3], we develop a predictor that adaptively learns interference patterns. Our design requires only an initial reception of pilot symbols used as input in a setup phase. This pilot scheme has been inspired by semi-blind channel estimation techniques [2], where a certain number of known pilot symbols are transmitted periodically for channel estimation and synchronization [11, 15, 17]. The pilot symbols help to estimate the expected reception power from the closest nodes. This power is used to construct a set of binary words, called transcodes. This set indicates the possible combinations of active interferers. In a learning phase, expected interference values accounting for contributions from nonclosest nodes are associated to each transcode. Finally, prediction is performed by combining these estimated transcode-interference pairs with the learning of the message durations of closest nodes. Using the current active interferers and the learnt traffic statistics, we predict the nodes that will remain active in future slots and thus the interference level to be expected. This interference prediction is used in the context of slot selection. Nodes attempt to select the most favorable starting slot for the transmission of a multislot message, from a set of slots, given an outage constraint. The performance is evaluated in terms of outage rate and delay; it is compared to a baseline scenario that selects slots uniformly at random.

Some related work is available: A cooperative interference prediction is used for link adaptation in [10]. Call admission control for DS-CDMA systems is done using intercell interference prediction in [7], and power control is achieved with interference prediction using a Kalman filter in [9]. A dynamical interference model is used in [1], where a mobility model is proposed to predict interference.

\section{NETWORK MODEL}

The nodes of a wireless network are distributed according to a Poisson point process (PPP) $\Phi$ with intensity $\lambda$ without mobility. A specific transmitter-receiver pair $(s, r)$ is considered for interference analysis (Fig. 1). Time is divided into slots. Medium access follows a slotted ALOHA-like scheme: every node $i$ generates messages of length $L_{i}$ slots by a Poisson arrival process with intensity $p$ and stores them in a queue. If the queue is not empty at the beginning of a new slot, the first message in the queue is transmitted within the next $\tau$ slots. The first slot of this message is determined by slot selection. The number of slots until this first slot is called delay $W$. A transmitting node is called active node. The message length $L_{i}$ of node $i$ is chosen from an exponential distribution with parameter $\eta$ rounded up to the next integer and remains at this value for this node. This yields a mean message length of $\mathbb{E}[L]=\frac{e^{\eta}}{e^{\eta}-1}$. The 


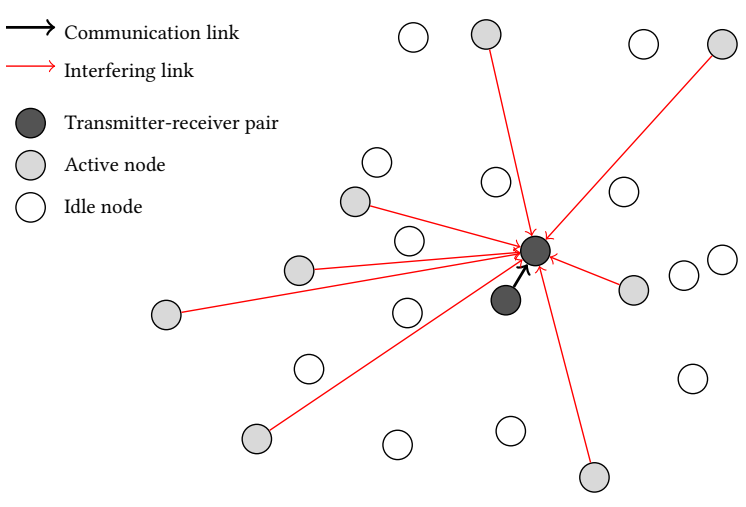

Figure 1: Network scenario.

expected fraction of nodes transmitting at a given slot is $p \mathbb{E}[L]$; values of $p$ and $\eta$ are chosen to ensure $p \mathbb{E}[L] \leq 1$.

A common wireless link model is used. The distance between $s$ and $r$ is called $d_{s r}$. The transmission power of each node is $\kappa$. The power attenuation over distance is modeled with a path gain

$$
\ell\left(d_{s r}\right)=\min \left(1,\left(\frac{d_{s r}}{d_{0}}\right)^{-\alpha}\right) .
$$

The path loss exponent $\alpha$ is constant across all slots, and reference distance $d_{0}$ needed for normalization is assumed to be $d_{0}=1 \mathrm{~m}$. The effects of multipath propagation are modeled by Nakagami- $m$ fading [12]. A block fading channel is assumed, in which the smallscale fading is independent in each slot and remains constant for the duration of a slot (as in [13]). The reception power arising from a transmission from $s$ to $r$ is $p_{r}(t)=\kappa \ell\left(d_{s r}\right) h_{s r}^{2}(t)$, where $h_{s r}(t)$ is Nakagami distributed. This transmission experiences co-channel interference from all other active nodes. The interference power at receiver $r$ in slot $t$ is

$$
I_{r}(t)=\sum_{u \in \Phi \backslash\{s\}} \kappa \ell\left(d_{u r}\right) h_{u r}^{2}(t) 1_{u}(t),
$$

where the indicator function $1_{u}(t) \in\{0,1\}$ states whether node $u$ is active (contributes to interference) in slot $t$ or not. The signal-tointerference ratio (SIR) is $\gamma_{r}(t)=\frac{p_{r}(t)}{I_{r}(t)}$. A link is in outage if and only if its SIR is below a certain threshold $\theta$ subject to the physical layer. The outage probability at $r$ in slot $t$ is $Q(t)=\mathbb{P}\left[\gamma_{r}(t)<\theta\right]=$ $1-\mathbb{P}\left[\gamma_{r}(t) \geq \theta\right]$, whose solution is given by [14, Theorem 3$]$.

The temporal dependance in (1) is caused by the fact that nodes are static (at unknown locations) and messages can be longer than one slot. This setup corresponds to Case $(2,1,2)$ in [13]. By learning the traffic patterns in the network, a transmitter-receiver pair should be able to predict its future interference situation and schedule transmissions to reduce outage. The following two sections describe how we do such prediction and timing adjustments.

\section{INTERFERENCE PREDICTION}

Setup Phase. Upon a setup request from node $r$ (or periodically), close-by nodes insert pilot symbols into their transmissions. Based on these pilots, $r$ estimates the average reception powers from its $k$ strongest interferers $u_{1}, u_{2}, \ldots, u_{k}$ and collects these values in a sorted way in a vector. The first vector element is the strongest interference value; the $k$ th is the weakest. The $k$-tuple of the indicator functions for the $k$ strongest interferers is the transcode $T(t)=\left(1_{u_{1}}(t), 1_{u_{2}}(t), \ldots, 1_{u_{k}}(t)\right)$. For each distinct transcode, $r$ adds up the estimated power values of the active nodes to yield its expected interference power. Overall, $2^{k}$ interference values are calculated and stored in a table, called $R x$-Table, together with their transcodes. Note that $k$ is a design parameter of the technique.

The setup phase is also used to estimate the Nakagami fading parameter $m$ for the link between receiver $r$ and its transmitter $s$. Methods proposed in $[8,18]$ can be applied to do so and get the estimate $\hat{m}$. We use maximum likelihood estimation in our work.

Learning Phase. Once the setup has been completed, all nodes transmit as usual. In order to learn, node $r$ measures the experienced interference in each slot. These measured values are compared to the interference values in the $R x$-Table. In each slot, we choose from the Rx-Table the transcode that corresponds to the largest interference value being smaller than the measured value. These estimated transcodes along with their measured interference values are stored for each slot $t$.

This data is used for two kinds of purpose at the end of the learning phase. First, $r$ estimates the message lengths $L_{i}$ and transmission probabilities $p_{i}$. This is done by analyzing the bit pattern in the estimations of the $1_{u_{i}}(t)$ for each of the $k$ interferers $u_{i}$. Second, all interference values with the same transcode are averaged. These average values are stored in an Int-Table together with their transcodes. Ideally, the learning phase is long enough to allow the Int-Table to contain all $2^{k}$ lines. If lines are missing, they will be taken from the Rx-Table. Both tables consist of $2^{k}$ lines and hence do neither introduce high memory nor high search cost for $k$-values of interest $(k \leq 5)$. The main difference between the two is that the Int-Table also accounts for the contribution of far away interferers.

Once the initial learning has been completed, the receiver enters the prediction phase. The Int-Table entries may be continuously refined while interference values keep being measured. This gives flexibility to the design by allowing to accommodate slow variations in the propagation scenario, arising e.g. from moving obstacles.

Prediction Phase. Node $r$ predicts in each slot $t$ for each of the $k$ strongest interferers whether this interferer will be active in the next slot $t+1$ or not. To do so, it uses a record of the $n$ most recently detected transcodes to predict the transcode $\hat{T}(t+1)$. It then gets the overall interference prediction $\hat{I}(t+1)$ for this transcode from the Int-Table. The value $n$ should be larger than the longest message of these interferers. Algorithm 1 summarizes the prediction. For each of the strongest interferers, the predictor checks if interferer $u_{i}$ is active in the current slot $t$. If not, the prediction will be that this interferer will remain inactive in slot $t+1$. If yes, the predictor counts as to how long the interferer has been transmitting. If this length is an integer multiple of the estimated message length $\hat{L}_{i}$ (acquired in the learning phase), the prediction is that the interferer will be inactive in slot $t+1$. Otherwise, the prediction is that it will remain active. Once this is done for all $k$ interferers, the predicted transcode $\hat{T}(t+1)$ is constructed, and the corresponding predicted interference $\hat{I}(t+1)$ is read from the Int-Table. 


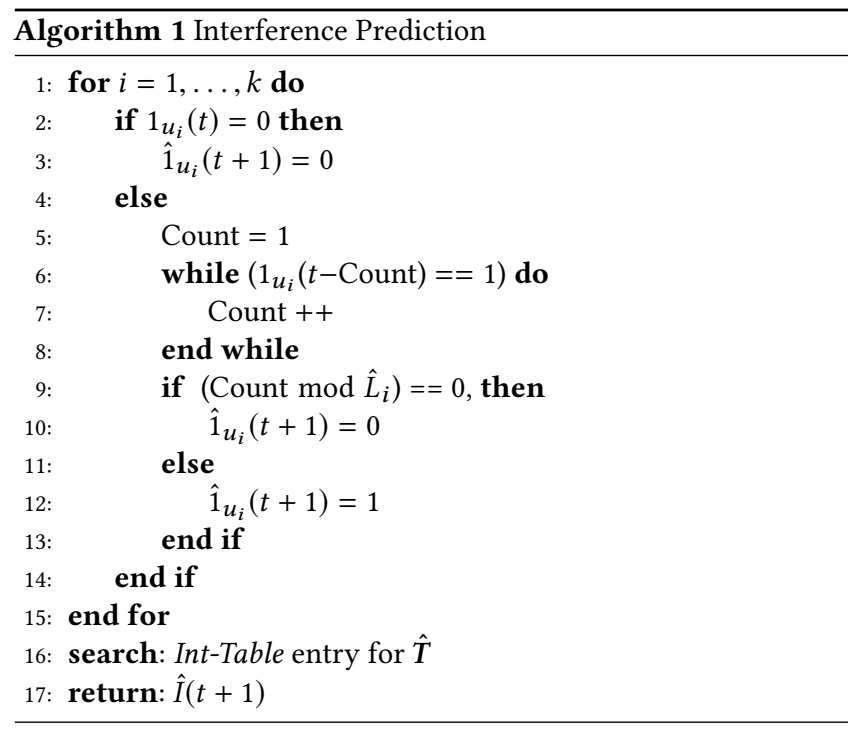

\section{SLOT SELECTION}

We utilize interference prediction to choose the best slot from $(\tau+1)$ following slots to start the transmission of a message. Our goal is to transmit as soon as possible constrained on an outage probability threshold $\xi$. This slot selection based on interference prediction

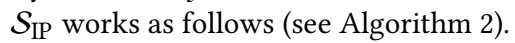

Node $r$ predicts the interference for the next slot using Algorithm 1 and uses $\hat{I}(t+1)$ to calculate the predicted outage $\hat{Q}(t+1)$. For Nakagami- $m$ fading with an estimated parameter $\hat{m}$, we have:

$$
\hat{Q}(t+1)=\frac{1}{\Gamma(\hat{m})} \gamma\left(\hat{m}, \frac{\theta \hat{I}(t+1)}{\ell\left(d_{s r}\right)} \hat{m}\right),
$$

with gamma function $\Gamma(\cdot)$ and incomplete gamma function $\gamma(\cdot, \cdot)$. Depending on the predicted outage and $\xi$, a decision on whether to transmit in slot $t+1$ is made. If $\hat{Q}(t+1) \leq \xi$, the predictor selects this slot. Otherwise, the decision is delayed by one slot. If no suitable slot is found within the transmission interval $\tau$, the last slot $t+\tau+1$ is chosen.

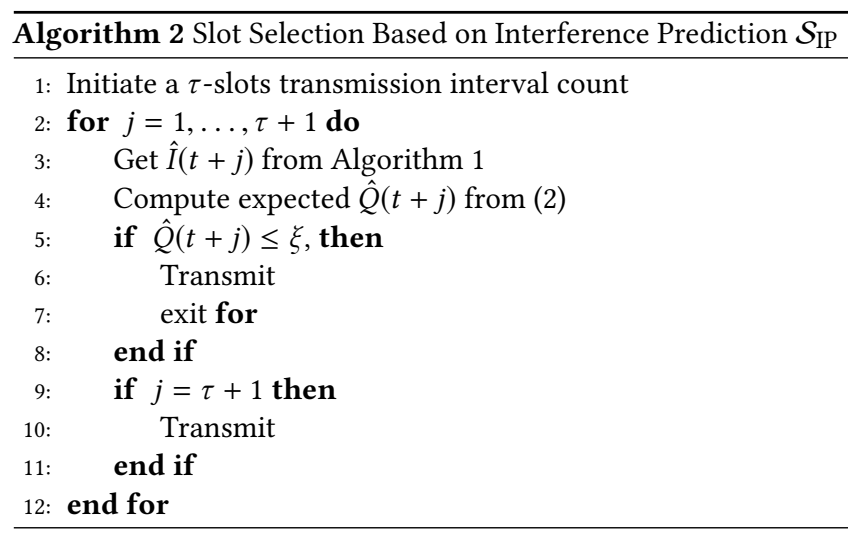

Low $\xi$ leads on average to high delay $W$, as the predicted outage $\hat{Q}(t+1)$ is unlikely to reach its target $\xi$ (and vice versa). It is important to note that lowering $\xi$ does not necessarily result in lower outage. This is because, at low $\xi$, the end of the transmission interval is reached very frequently. The node is then forced to transmit in the last slot even if the threshold is not met; it thus missed an earlier slot with lower outage probability.

\section{NUMERICAL EVALUATION}

To investigate the performance achieved with $\mathcal{S}_{\text {IP }}$, we simulate a network with node density $\lambda$ in a square area of size $A=10,000 \mathrm{~m}^{2}$. Due to the stationarity of the PPP and Slivnyak's theorem [5], we can assume that $r$ is located in the middle of the area. Each other node transmits as described in Section 2 with parameters $p=0.1$ and $\mathbb{E}[L]=5$ using a fixed transmit power $\kappa=1 \mathrm{~mW}$. The path loss exponent is $\alpha=3$. We compare $\mathcal{S}_{\text {IP }}$ with random slot selection $\mathcal{S}_{\text {Rand }}$, which choses the first slot randomly from a uniform distribution between 0 and $\tau$. Both selection schemes use the same $\tau$. Simulations are performed over 20,000 slots with a learning phase of 1,000 slots and are averaged over 10,000 independent realizations of $\Phi$. All simulations are made using the $\mathrm{R}$ project for statistical computing.

The proper functioning of interference prediction is illustrated in Fig. 2, which compares the predicted and measured interference for 100 slots in a single realization of $\Phi$. The predicted interference is close to the true interference for most slots with a mean square error of MSE $=0.068(\mathrm{~mW})^{2}$. Deviations are mainly caused by fading and interference from some close nodes starting new transmissions.

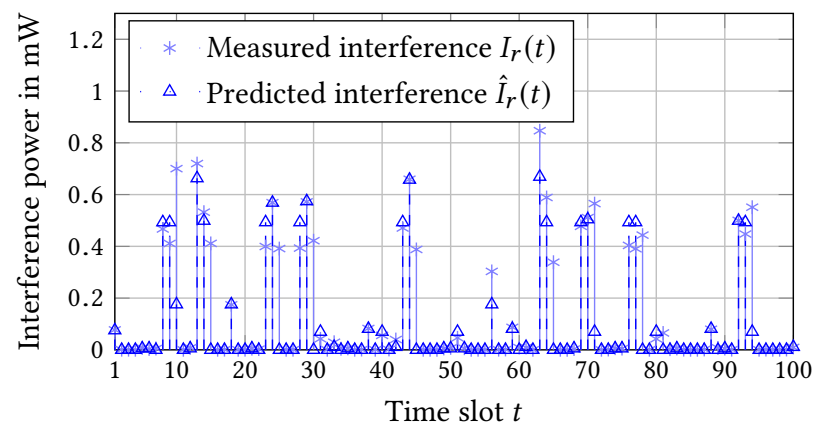

Figure 2: Measured and predicted interference power values

Impact of Node Density on Outage: Fig. 3 shows the impact of $\lambda$ on outage. $\mathcal{S}_{\text {IP }}$ has a lower outage rate than $\mathcal{S}_{\text {Rand }}$ for all $\lambda$-values. The relative difference decreases with increasing $\lambda$ because if nodes are far apart (sparse network, low $\lambda$ ), the few closest nodes can be easily distinguished, leading to good interference prediction; if nodes are close together (dense network, high $\lambda$ ), the distinction between closest neighbors degrades, resulting in poor interference prediction and consequently a smaller benefit in terms of outage.

Impact of Mean Delay on Outage: Fig. 4 shows the outage over the mean delay $\bar{W}$ for $\mathcal{S}_{\text {IP }}$. Since delay cannot be controlled directly, we vary $\xi$ from 0 (high delay expected) to 1 (low delay expected). For random slot selection, the mean delay is always $\bar{W}=\tau / 2-$ the line just indicates the outage rate for comparison. For reference, we show the results of $\mathcal{S}_{\mathrm{IP}}$ fed with true interference values to make perfect slot selection. The main result is that $\mathcal{S}_{\text {IP }}$ always outperforms $\mathcal{S}_{\text {Rand }}$ in terms of outage (except for the extreme cases $\bar{W}=0$ and $\tau$, where all have the same outage because $\mathcal{S}_{\text {IP }}$ always 


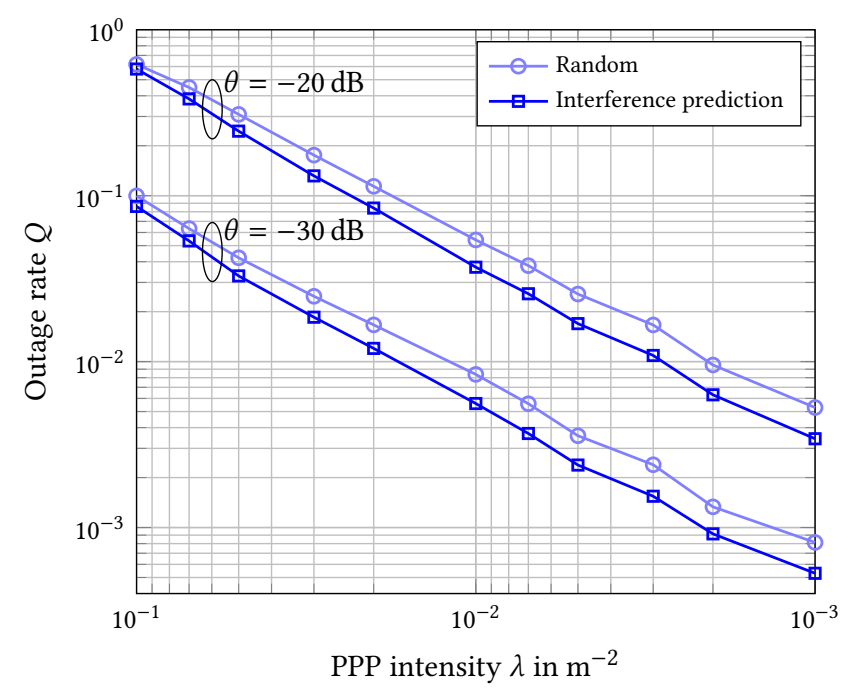

Figure 3: Outage over PPP intensity $\lambda$ with $m=3.5, p=0.09$, $\tau=6, k=5$, and $\mathbb{E}[L]=5.5$. We use here a larger area of $A=1 \mathbf{k m}^{2}$ to have enough active nodes for low $\lambda$.

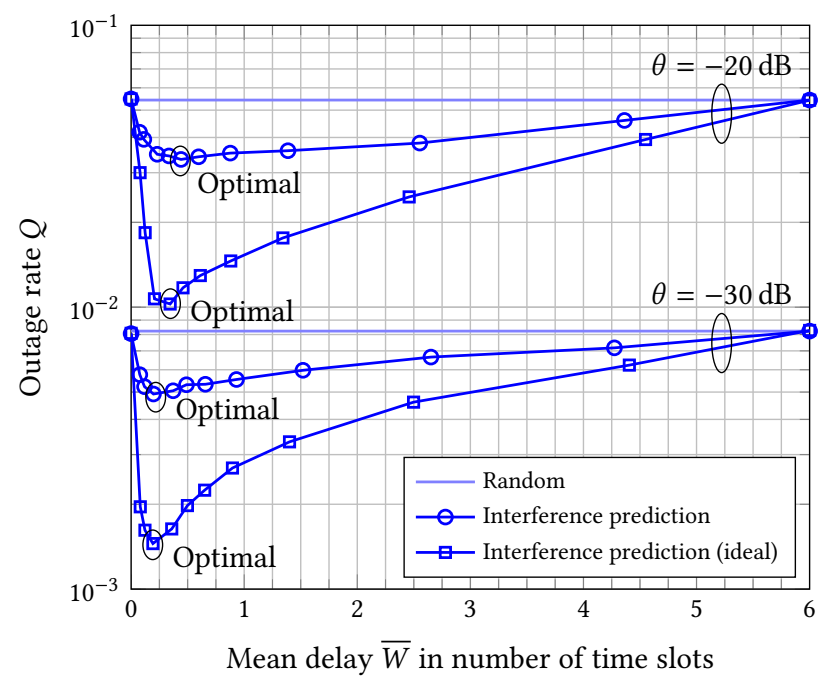

Figure 4: Outage over mean delay $\bar{W}$ with $m=3.5, \tau=6$, $\lambda=10^{-2}, p=0.1, k=5$, and $\mathbb{E}[L]=5$.

chooses the first or last slot, respectively). Note that an optimal operation point exists, which will be used in the following.

Impact of Transmission Interval on Delay: Fig. 5 shows that the mean delay of $\mathcal{S}_{\mathrm{IP}}$ is lower than that using $\mathcal{S}_{\text {Rand }}$ for all values of $\tau$ (except for $\tau=0$, where both have zero delay). The mean delay using interference prediction increases with increasing $\tau$ over the full range of the plot (which shows that $\mathcal{S}_{\text {IP }}$ sometimes uses the entire transmission interval to find a suitable slot). Nevertheless, this increase is much slower than the one using $\mathcal{S}_{\text {Rand }}$.

Impact of Message Length on Outage: Fig. 6 studies the impact of $\mathbb{E}[L]$ on outage. We vary $\mathbb{E}[L]$ but keep $p \mathbb{E}[L]$ constant (at 0.5 ), thus

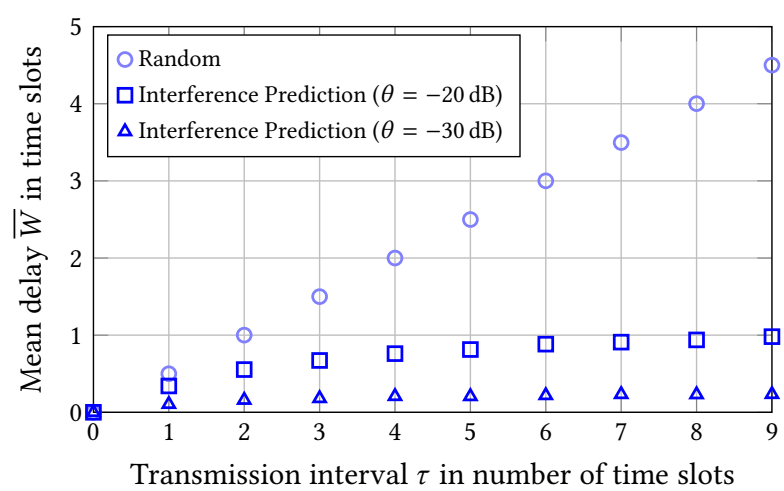

Figure 5: Mean delay over transmission interval $\tau$ with $m=$ $3.5, \lambda=10^{-2}, p=0.1, k=5$, and $\mathbb{E}[L]=5$.

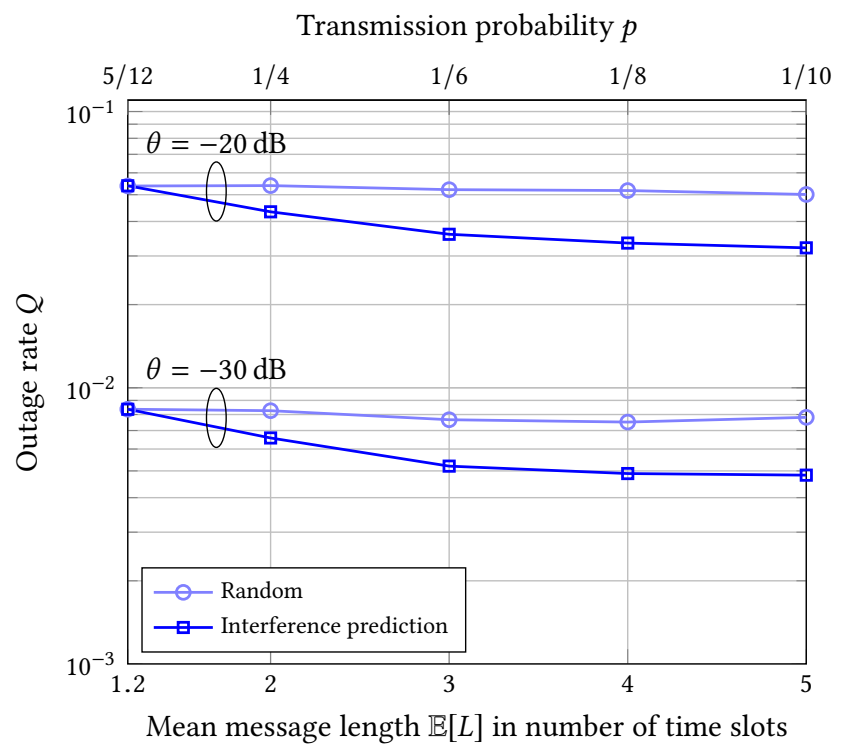

Figure 6: Outage over mean message length $\mathbb{E}[L]$ with $m=3.5$, $\lambda=10^{-2}, k=5$, and $\tau=9$

having on average a constant fraction of active nodes and hence constant interference. The benefit of interference prediction is good for long messages but low for short ones. As short messages go along with high $p$, the technique is unable to predict nodes that become active in the next slot; the predictor underestimates the interference, resulting in outage close to random slot selection. Long messages, in turn, help the interference prediction.

Impact of Fading on Outage and Delay: To analyze the sensitivity of $\mathcal{S}_{\text {IP }}$ against fading, we evaluate the outage rate for different values of the Nakagami parameter $m$ (Fig. 7). Recall that the severeness of fading increases with decreasing $m$. As expected even without interference, the outage rate decreases for increasing $m$.

Two types of $\mathcal{S}_{\text {IP }}$ are used: First, we optimize $\xi$ individually for each $m$ in terms of outage, where this optimal $\xi$ is obtained by simulations (recall Fig. 4). Second, since the optimal $\xi$ depends on 


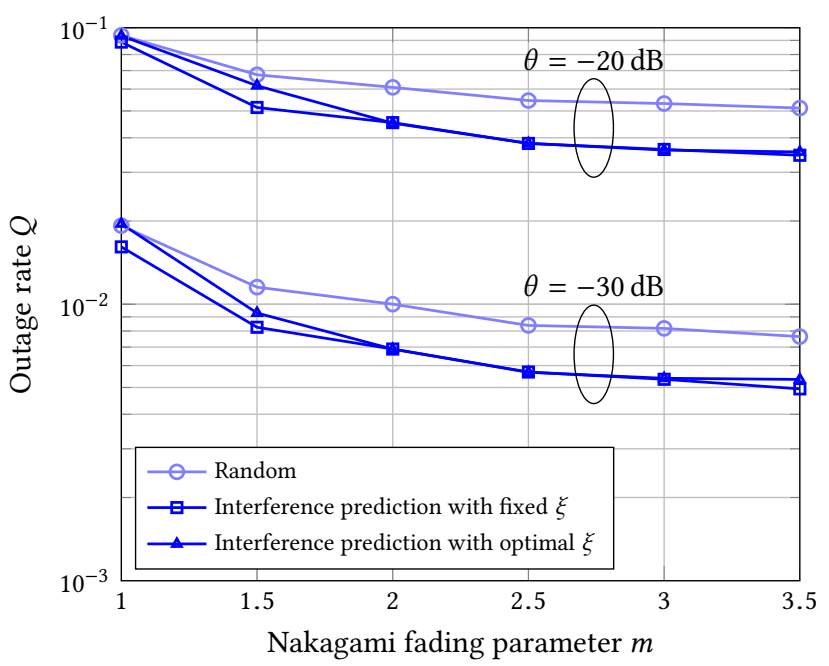

Figure 7: Outage over Nakagami fading parameter $m$ with $\lambda=10^{-2}, p=0.1, k=5, \tau=6$, and $\mathbb{E}[L]=5$.

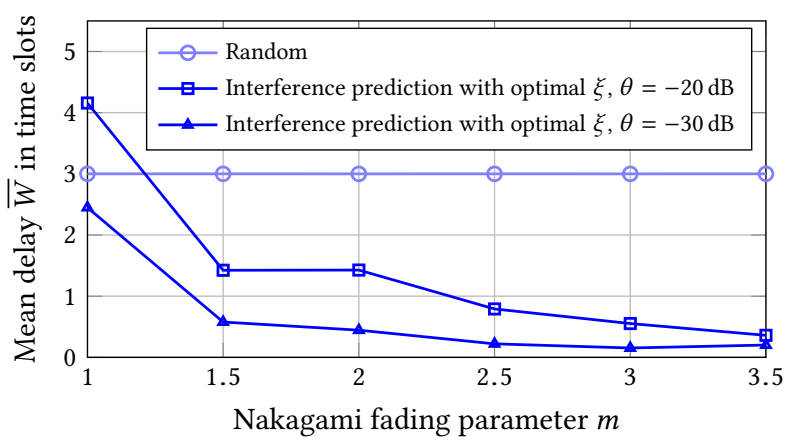

Figure 8: Mean delay over Nakagami fading parameter $m$ with $\lambda=10^{-2}, p=0.1, k=5, \tau=6$, and $\mathbb{E}[L]=5$.

many parameters and takes effort to be determined, we keep $\xi$ constant over all $m$ (we set $\xi$ to its optimal value for $m=2$ ). As expected, the first approach yields a lower outage rate than the second one. The difference is significant for strong fading (low $m$ ) but becomes negligible for weak fading (high $m$ ).

Both $\mathcal{S}_{\text {IP }}$ types outperform $\mathcal{S}_{\text {Rand }}$ independent of the severeness of the fading - the actual gain depends on $m$ - meaning that the proposed slot selection scheme is somehow robust against fading. However, recall that the MSE in the actual interference prediction significantly depends on $m$. This is related to the predictor design, as it does not make use of fading channel estimation.

Finally, Fig. 8 shows the mean delay over $m$ for optimally chosen $\xi . \mathcal{S}_{\text {IP }}$ has shorter mean delays than $\mathcal{S}_{\text {Rand }}$ for almost all $m$. Only when $m$ is close to 1 , the delay increases, as the interference prediction degrades significantly.

\section{CONCLUSIONS AND OUTLOOK}

Slot selection based on a simple interference prediction technique performs well in certain scenarios, especially in sparse networks with long messages. The gains in outage and delay show the potential of interference prediction as a building block in wireless systems. The performance strongly depends, however, on a proper choice of the outage threshold $\xi$. The proposed technique underestimates interference because transcode estimation does not consider newly started transmissions. It is expected that advanced learning techniques will improve interference prediction (probably at the cost of increased complexity). Further work is needed to design such advanced techniques, discuss their integration into the protocol stack, and assess their practicability in real systems. Comparisons with related interference management concepts, such as carrier sense medium access with backoff, must be made.

\section{ACKNOWLEDGMENTS}

This work was supported by grant P24480-N15 of the Austrian Science Fund (FWF) and grant 20214/26481/38805 of the KWF.

\section{REFERENCES}

[1] Yirui Cong, Xiangyun Zhou, and Rodney A. Kennedy. 2015. Interference prediction in mobile ad hoc networks with a general mobility model. IEEE Trans. Wireless Commun. 14, 8 (2015), 4277-4290.

[2] Elisabeth D. Carvalho and Dirk T. M. Slock. 1997. Cramer-Rao bounds for semiblind, blind and training sequence based channel estimation. In Proc. Workshop on Signal Process. Adv. in Wireless Commun. IEEE, Paris, France, 129-132.

[3] Radha K. Ganti and Martin Haenggi. 2009. Spatial and temporal correlation of the interference in ALOHA ad hoc networks. IEEE Commun. Lett. 13, 9 (2009), 631-633.

[4] Martin Haenggi. 2009. Outage, local throughput, and capacity of random wireless networks. IEEE Trans. Wireless Commun. 8, 8 (2009), 4350-4359.

[5] Martin Haenggi. 2013. Stochastic Geometry for Wireless Networks. Cambridge University Press, Cambridge, United Kingdom.

[6] Martin Haenggi and Roxana Smarandache. 2013. Diversity polynomials for the analysis of temporal correlations in wireless networks. IEEE Trans. Wireless Commun. 12, 11 (2013), 5940-5951.

[7] Il M. Kim, Byung C. Shin, and Dong J. Lee. 2000. SIR-based call admission control by intercell interference prediction for DS-CDMA systems. IEEE Commun. Lett. 4, 1 (2000), 29-31.

[8] Young C. Ko and Mohamed S. Alouini. 2003. Estimation of Nakagami- $m$ fading channel parameters with application to optimized transmitter diversity systems. IEEE Trans. Wireless Commun. 2, 2 (2003), 250-259.

[9] Kin K. Leung. 2002. Power control by interference prediction for broadband wireless packet networks. IEEE Trans. Wireless Commun. 1, 2 (2002), 256-265.

[10] Andreas Müller and Philipp Frank. 2010. Cooperative interference prediction for enhanced link adaptation in the 3GPP LTE uplink. In Proc. Vehicular Technology Conf. (VTC). IEEE, Taipei, Taiwan.

[11] Bertrand Muquet, Marc D. Courville, and Pierre Duhamel. 2002. Subspace-based blind and semi-blind channel estimation for OFDM systems. IEEE Trans. Signal Process. 50, 7 (2002), 1699-1712.

[12] Minoru Nakagami. 1958. The $m$-distribution - A general formula of intensity distribution of rapid fading. In Proc. Symp. on Statistical Methods in Radio Wave Propag. Pergamon Press, Los Angeles, USA, 3-36.

[13] Udo Schilcher, Christian Bettstetter, and Günther Brandner. 2012. Temporal correlation of interference in wireless networks with Rayleigh block fading. IEEE Trans. Mobile Comput. 11, 12 (2012), 2109-2120.

[14] Udo Schilcher, Stavros Toumpis, Martin Haenggi, Alessandro Crismani, Günther Brandner, and Christian Bettstetter. 2016. Interference functionals in Poisson networks. IEEE Trans. Inf. Theory 62, 1 (2016), 370-383.

[15] Arnold L. Swindlehurst and Geert Leus. 2002. Blind and semi-blind equalization for generalized space-time block codes. IEEE Trans. Signal Process. 50, 10 (2002), 2489-2498.

[16] Ralph Tanbourgi, Harpreet S. Dhillon, Jeffrey G. Andrews, and Friedrich K. Jondral. 2014. Effect of spatial interference correlation on the performance of maximum ratio combining. IEEE Trans. Wireless Commun. 13, 6 (2014), 33073316.

[17] Fredrik Tufvesson and Torleiv Maseng. 1997. Pilot assisted channel estimation for OFDM in mobile cellular systems. In Proc. Vehicular Technology Conf. (VTC). IEEE, Phoenix, AZ, USA, 1639-1643.

[18] Qi T. Zhang. 2002. A note on the estimation of Nakagami- $m$ fading parameter. IEEE Commun. Lett. 6, 6 (2002), 237-238. 